\title{
Low-Force DNA Condensation and Discontinuous High-Force Decondensation Reveal a Loop-Stabilizing Function of the Protein Fis
}

\author{
Dunja Skoko, ${ }^{1}$ Jie Yan, ${ }^{2}$ Reid C. Johnson, ${ }^{3}$ and John F. Marko ${ }^{1}$ \\ ${ }^{1}$ Department of Physics, University of Illinois at Chicago, 845 West Taylor Street, Chicago, Illinois 60607-7059, USA \\ ${ }^{2}$ Department of Physics, National University of Singapore, Singapore 117542 \\ ${ }^{3}$ Department of Biological Chemistry, David Geffen School of Medicine at UCLA, Los Angeles, California 90095-1737, USA
}

(Received 7 May 2005; published 8 November 2005)

\begin{abstract}
We report single-DNA-stretching experiments showing that the protein Fis, an abundant bacterial chromosome protein of $E$. coli, mediates a dramatic DNA condensation to zero length. This condensation occurs abruptly when DNA tension is reduced below a protein-concentration-dependent threshold $f^{*}<$ $1 \mathrm{pN}$. Following condensation, reopening under larger forces proceeds via a series of discrete jumps, indicating that Fis is able to stabilize DNA crossings. Our experiments suggest that Fis may play a role in vivo stabilizing the "loop-domain" structure of the bacterial chromosome.
\end{abstract}

Fis is a member of a small set of abundant proteins associated with the chromosome of $E$. coli and related bacteria [1]. Fis molecules form dimers which bind to and bend DNA in a "nonspecific" (sequence-independent) fashion. In addition, Fis selectively binds to particular sequences to regulate activities of other DNA-binding proteins, including recombinases and polymerases. Strikingly, Fis concentration in vivo increases with bacterial growth rate [2]; during nutrient-rich rapid growth Fis is the most abundant DNA-binding protein in $E$. coli with up to 40000 dimers per cell, i.e., $\approx 50 \mu \mathrm{M}$ concentration. These properties suggested that Fis might have an important function in organizing the bacterial chromosome, motivating us to look at its effects on individual DNA double helix molecules.

We report single-DNA experiments revealing two effects of Fis on DNA. For forces $>1 \mathrm{pN}$, Fis-DNA complexes display reversible elasticity similar to "naked" DNA, but shifted to higher forces, in accord with experiments and theories for effects of DNA-bending proteins $[3,4]$. However, for Fis concentrations $c>1 \mu \mathrm{M}$, we find that below a concentration-dependent sub-piconewton threshold force Fis-coated DNA undergoes drastic condensation to zero length; subsequently, higher forces of roughly $10 \mathrm{pN}$ reopen the DNA via discontinuous jumps. These effects indicate that Fis is able to stabilize DNA crossings and therefore may play a role in stabilizing the loop-domain structure of the bacterial chromosome [5,6]. Our experiments, while similar in some respects to a study of DNA-condensing multivalent salts and polypeptides [7], are distinct in their observation of slow DNA condensation via stabilization of nonspecific DNA crossings by protein.

We tethered $48.5 \mathrm{~kb} \lambda$-DNA molecules by one end to a glass cover slide, and at the other end to a paramagnetic bead, to which force was applied using a magnetic field gradient [8] (for method see Ref. [9]). Such "magnetic tweezer" setups permit monitoring of protein interactions under constant DNA tension [10,11]. Following force measurements in protein-free buffer $(20 \mathrm{mM}$ HEPES,

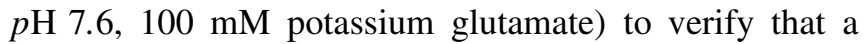
bead was tethered by a single DNA of expected length of $16.5 \mu \mathrm{m}[12,13]$, a "high force" of $14 \mathrm{pN}$ was applied, and buffer containing $13 \mu \mathrm{M}$ Fis was introduced.

Upon Fis binding at $14 \mathrm{pN}$, DNA extension decreased by $\approx 1 \mu \mathrm{m}$; then as force was reduced to $\approx 1 \mathrm{pN}$, the Fisbound DNA gradually became less extended [Fig. 1(a)], with reversible elastic response (no hysteresis) and extension shorter than that of naked DNA at each force. These

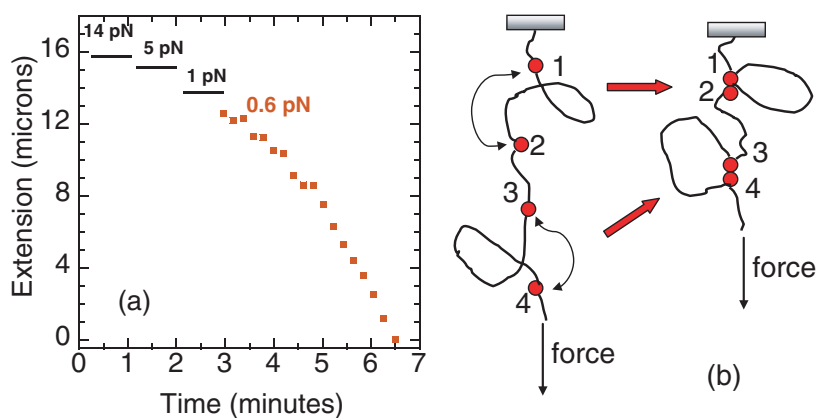

FIG. 1 (color). (a) Collapse of DNA against applied force by Fis. After incubation with $13 \mu \mathrm{M}$ Fis in buffer (20 mM HEPES, $100 \mathrm{mM}$ potassium glutamate, $p \mathrm{H} \mathrm{7.6)}$ at a force of $14 \mathrm{pN}$, DNA extension decreased from 16.5 to $15.5 \mu \mathrm{m}$, consistent with the action of a DNA-bending protein. As force was subsequently reduced to $5 \mathrm{pN}$ and later to $1 \mathrm{pN}$, DNA length decreased over a few seconds to stable final values (horizontal black lines), expected given the known DNA-bending function of Fis. By contrast, at $0.6 \mathrm{pN}$ force, the DNA-Fis complex condensed (red squares); between 3 and 7 min DNA was gradually compacted down to zero extension. At this force, naked DNA extension would be $14.5 \mu \mathrm{m}$. (b) Proposed model for DNA condensation by Fis against applied force. Thermally excited DNA loops can be stabilized by Fis molecules (red circles). Fis likely coats the DNA at concentrations where looping is observed; the red circles show only those complexes capable of stabilizing loops. 
effects are in accord with experiments on other DNAbending proteins $[3,9,14]$.

However, when force on the $13 \mu \mathrm{M}$ Fis-DNA complex was reduced to $0.6 \mathrm{pN}$, a qualitatively new behavior was observed. Over the next 3.5 min the Fis-DNA complex dramatically condensed; extension shrunk from $13 \mu \mathrm{m}$ down to zero [Fig. 1(a), red squares]. We will refer to the force at which this condensation occurs as the "threshold force" $f^{*}$; this abrupt condensation at low $(<1 \mathrm{pN})$ forces suggests Fis is able to stabilize DNA crossings [Fig. 1(b)].

In a series of experiments, we found $f^{*}$ to be Fis concentration dependent [Fig. 2(a)]; binding of Fis to DNA at 1,6 , and $13 \mu \mathrm{M}$ concentrations led to $f^{*}=0.2,0.3$, and $0.6 \mathrm{pN}$, respectively, with $f^{*}$ values reproduced in separate experiments to $0.1 \mathrm{pN}$. Notably, condensation was more rapid for the lower threshold forces $f^{*}$ obtained at lower Fis concentrations. Finally, at $200 \mathrm{nM}$ Fis, we no longer observed DNA condensation at any force above our lower force limit $(0.05 \mathrm{pN})$. In bulk phase gel mobility shift experiments Fis associates nonspecifically with DNA

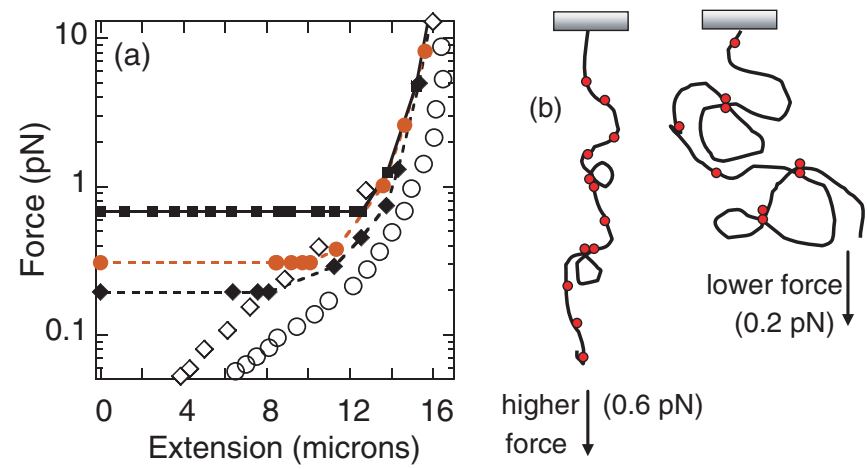

FIG. 2 (color). Force-extension behavior of Fis-DNA complexes. (a) Open circles indicate naked DNA in buffer: as in other studies, a nonlinear and reversible polymer elastic response is observed. Squares show DNA after reaction with $13 \mu \mathrm{M}$ Fis at a force of $14 \mathrm{pN}$. Fis-induced DNA bends result in shortening of the DNA length from 16.5 to $15.5 \mu \mathrm{m}$; for forces above $0.6 \mathrm{pN}$ (extensions above $12.5 \mu \mathrm{m}$ ) the force-extension is shifted to slightly larger forces, and is reversible. However, at the threshold force of $0.6 \mathrm{pN}$ abrupt DNA condensation occurs. In other experiments with $6 \mu \mathrm{M}$ (solid circles) and $1 \mu \mathrm{M}$ Fis (solid diamonds), abrupt condensation was again observed, with the difference that at lower protein concentrations the threshold force is gradually shifted down towards smaller forces. At or below $200 \mathrm{nM}$ Fis concentration, abrupt condensation no longer occurs (open diamonds) and the Fis-DNA complex displays reversible elasticity over the entire force range. Note that data points for forces above collapse represent averages of stable series of extension measurements. (b) Relation between Fis concentration and threshold force $f^{*}$. At high protein concentration (left) loop-forming complexes are in close proximity, allowing small, thermally excited loops to be captured; these loops form at relatively high forces. At lower concentrations (right) loop-forming sites are further apart, so condensation can only occur via formation of larger loops; these loops form only at relatively low forces. such that initially bound complexes form at low nanomolar concentrations; the molecules become nearly fully coated by $50 \mathrm{nM}[15,16]$. Thus, the experiments in this Letter are all well into the saturated-binding regime.

The high-force, reversible force-extension behaviors of DNA with Fis bound at $0.2,1,6$, and $13 \mu \mathrm{M}$ were nearly the same: each displayed a similar modest compaction, due to DNA bending [Fig. 2(a)]. We conclude that above $200 \mathrm{nM}$ Fis concentrations, the DNA is entirely coated by Fis, leading to a saturation of its DNA-bending function. The force shifts are consistent with those expected from a protein which deflects DNA by $\approx 40^{\circ}$ (the bend expected for Fis) once every $\approx 7 \mathrm{~nm}$ [4] (roughly the minimal spacing for Fis dimers bound consecutively along a DNA). The high-force reversible force-extension curves, when fit to the corresponding result for the semiflexible polymer for extensions from $60 \%$ to $90 \%$ full length [12] yield an effective persistence length of $20 \pm 6 \mathrm{~nm}$ (4 measurements), about half that of naked DNA in our buffer $(45 \pm 4 \mathrm{~nm})$.

The dependence of $f^{*}$ on protein concentration, as well as their low $(<1 \mathrm{pN})$ values suggested to us that the condensation occurs via Fis stabilizing DNA self-crossings
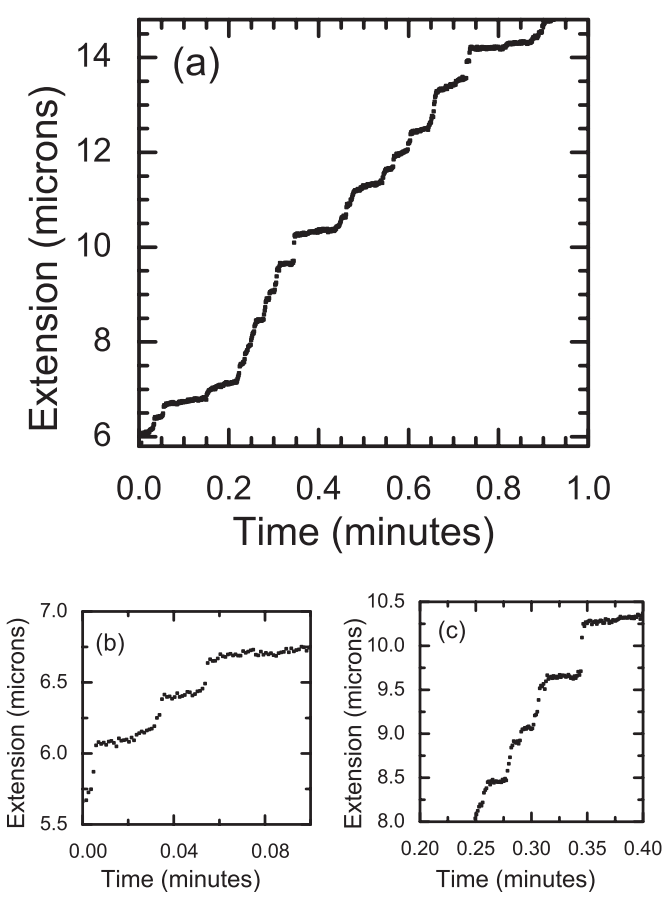

FIG. 3. Decondensation of Fis-condensed DNA shows discrete jump opening events, consistent with opening of Fis-mediated DNA loops. Extension versus time recorded during the opening driven by $9 \mathrm{pN}$ force, following 3 min condensation by $5 \mu \mathrm{M}$ Fis at $<0.2 \mathrm{pN}$ (note condensation reaction is not shown). (a) Entire opening time series shows opening of molecule from near zero to near the full contour length of $16.5 \mu \mathrm{m}$. Note that the small discontinuities between points correspond to small jump events. (b) Expanded view of first 0.1 min of (a) shows discrete extension jumps more clearly. (c) Expanded view of later part of (a) shows similar jumps. 
or "loops" generated by thermal fluctuation [Fig. 1(b)]. Loops along a DNA under tension are expected to be strongly suppressed above $1 \mathrm{pN}[17,18]$, but will form at lower forces where conformational fluctuations become likely. If, at low enough forces, the rate of loop formation becomes large enough to exceed the rate that loops open, condensation would occur. This loop-stabilization hypothesis suggested that following Fis-condensation of DNA, an increase of force to well above $f^{*}$ should lead to a series of discrete jumps in extension corresponding to the opening of individual Fis-stabilized DNA crossings. To test this we used a different "transverse" magnetic tweezer setup with finer position and time resolution [19]. Fis $(5 \mu \mathrm{M})$ was bound to DNA, and the complex condensed after force was reduced $<0.3 \mathrm{pN}$. Upon application of a high force $(9 \mathrm{pN})$, the DNA extended via a series of discrete jumps (Fig. 3). The jump sizes are distributed from $40 \mathrm{~nm}(120 \mathrm{bp})$, the minimum jump length we can reliably measure above thermal noise, to $200 \mathrm{~nm}$ (600 bp). The majority of the jumps are between 40 and $60 \mathrm{~nm}$ (120 to $180 \mathrm{bp}$ ) in size.

We note that following forced opening of Fis-condensed DNA, recondensation could be repeated by reducing $f<$ $f^{*}$, even after washing protein out of the experimental chamber, indicating that the opening by force does not irreparably damage Fis or drive it off DNA. Finally, opening of Fis-condensed DNA by force always proceeds via jumps, distinct from the case of polycations studied by Baumann et al. [7] where a rather smooth force plateau with no jumps was often found. We note that for Fis, the precise series of jump heights is different in each opening, as is to be expected given closure of the molecule by random thermal motion.

Our data therefore indicate that Fis has two separate structural effects on DNA. At low concentrations $(\leq 200 \mathrm{nM})$, Fis bends DNA. However, $\geq 1 \mu \mathrm{M}$ Fis leads to DNA-loop-stabilizing complexes, which are able to condense DNA for forces below a concentrationdependent threshold force. The loop-stabilizing function explains previous electron and atomic force microscope studies where Fis was observed to enhance branching (i.e., crossings) in supercoiled plasmids [20].

Our observations can be understood considering the free energy of the loops formed:

$$
F=F_{\text {loop }}(l)+f l-k_{B} T n \ln \frac{c}{K} .
$$

The first term, $F_{\text {loop }}(l)$, is the cost of bringing the ends of an unstretched polymer segment of contour length $l$ to within $1 \mathrm{~nm}$ (essentially the logarithm of the " $J$-factor" probability for polymer cyclization [21]). $F_{\text {loop }}$ diverges for small $l$ for energetic reasons and for large $l$ for entropic reasons, and has a minimum value $\approx 12 k_{B} T$ for $l \approx 3.5$ persistence lengths [18]. The second term $f l$ accounts for the additional work that must be done against the force to form the loop (this form is for a fully stretched polymer; correction for conformational fluctuation [12] is easily included, see [17]). The force $f$ acts to increase the total barrier to loop formation, to $F_{\text {loop }}+f l$; this free energy cost still has a single minimum.

The third term of Eq. (1) is the "binding" free energy that stabilizes the loop; this term should increase with bulk protein concentration $c$. The $\ln c$ dependence written in Eq. (1) is appropriate for independent and reversible binding of loop-forming complexes, with a dissociation constant $K$, and where $n$ is the number of DNA-bound Fis dimers that must combine together to anchor one loop. While in principle $K$ depends on the applied force as $\approx$ $K_{0} e^{b f / k_{B} T}$ where $b$ is a $\approx 1 \mathrm{~nm}$ distance describing the range of the chemical interactions stabilizing the DNAprotein complex [22], this introduces a small correction to Eq. (1) since $b \ll l$; this is neglected here.

The barrier and binding terms of Eq. (1) compete; the sign of $F$ determines loop stability. For sufficiently large $f$, $F(l)>0$ for all $l$; all loops are unstable. Since $F_{\text {loop }}+f l$ has a minimum, as force is reduced $F=0$ will occur first at that minimum, for some loop size $l^{*}$ (note $l^{*}$ is forcedependent). The threshold force $f^{*}$ at which these loops become stable is:

$$
f^{*}=\frac{k_{B} T}{l^{*}}\left[n \ln \frac{c}{K}-\frac{F_{\text {loop }}\left(l^{*}\right)}{k_{B} T}\right] .
$$

For sufficiently small protein concentration $c, f^{*}<0$ : loops are never stable. However, for $c$ sufficiently large, $f^{*}>0$ : loops are stable at that concentration for forces less than $f^{*}$.

Equation (2) indicates $f^{*}$ should have a weak dependence on bulk protein concentration $c$, similar to that observed experimentally ( $>$ tenfold increase in $c$ leads to only a factor of 3 change in $f^{*}$ ). A linear fit of $f^{*}$ to $\ln c$ gives $n=1.8 \pm 0.4$, consistent with two Fis molecules stabilizing each crossing. Equation (1) also qualitatively explains the increase of reaction rate as $f^{*}$ decreases, since the loop-closing barrier, $F_{\text {loop }}(l)+f^{*} l$, decreases as $f^{*}$ decreases. Finally, the force scale for condensation reaction is $\approx k_{B} T / l^{*}$, where $l^{*} \approx 20 \mathrm{~nm}$ (recall that Fis reduces the effective persistence length by about a factor of 2 via its bending of DNA, plus $l^{*}$ is shifted to lower values by the applied force [18]). This roughly explains the low forces at which we observe Fis condensation of DNA, in comparison to the few $\mathrm{pN}$ force threshold observed in experiments where DNA is tightly condensed by salts or ligands [7].

Whereas illustrative of the basic physics of loopingdriven DNA condensation, Eq. (1) neglects cooperativity between loops, which can arise simply via Fis stabilization of interloop crossings. The sharp force threshold and strong hysteresis observed after collapse in our experiment $(<1 \mathrm{pN}$ required for condensation, $>5 \mathrm{pN}$ required for opening) both indicate that cooperativity is an important ingredient. A recent theory of loop-mediated DNA condensation [23] indicates that our experiments are compatible with a binding model including cooperativity, and also 
predicts a shift of the distribution of loops to smaller sizes with increasing critical force.

In further experiments, after reacting DNA with $5 \mu \mathrm{M}$ Fis (sufficient concentration for looping-condensation to occur), we added buffer containing free DNA $(0.05 \mathrm{mg} / \mathrm{ml}$ $300 \mathrm{bp}$ fragments); the result was that looping condensation no longer occurred. However, Fis was still present along the DNA as evidenced by the high-force shift (data not shown). Also, we found sufficiently high salt concentration (300 $\mathrm{mM}$ potassium glutamate) eliminated looping condensation, but again the bending-driven shift of the force-extension curve remained (data not shown). These results provide additional evidence that DNA bending and loop stabilization are distinct functions, possibly accomplished by Fis binding to DNA in different ways. Bimodal behavior of DNA-binding proteins has also been observed for IHF [22], in a concentration-dependent manner for $\mathrm{HU}$ $[9,14]$, and also for HMGB1/2 $[9,24]$; possibly this is a common feature of protein-DNA interactions.

Current evidence indicates that bacterial chromosomes are divided into topologically isolated domains or loops, averaging about $10 \mathrm{~kb}$ in rapidly growing cells, but of larger size in stationary phase $[5,6,25]$. DNA-binding proteins such as H-NS, SeqA, MukBEF, and topoisomerases are suspected to contribute to domain stabilization [5]. The results of this Letter, combined with the strong correlation of in vivo Fis levels with growth rate, suggest that Fis may also play an important role in generating the large number of chromosomal domains within the bacterial nucleoid. Formation of DNA loops by Fis within promoter regions also has been proposed to potentiate transcription [26].

Although Fis in rapidly growing $E$. coli is present at concentrations well above the $1 \mu \mathrm{M}$ needed to support looping in our single-DNA reactions, the number of Fis dimers averages less than one every $200 \mathrm{bp}$ of chromosomal DNA. Thus, levels of Fis, or any of the other abundant nucleoid proteins, are insufficient to globally coat chromosomal DNA in vivo [1]. Fis is known to preferentially bind to specific sites that display high sequence degeneracy; the $\lambda$-DNA used in this study contains a few such sites within its regulatory regions $[15,27]$ in a manner similar to the distribution of specific Fis binding sites in the E. coli chromosome. High-affinity Fis binding sites can be clustered within intergenic regions and might nucleate localized high densities of DNA-bound Fis dimers [1]. Moreover, the interwound and branched structure of the plectonemically supercoiled bacterial chromosome, combined with cytoplasmic crowding forces, will encourage interactions between remote Fis-bound segments in vivo [28]. Effects of specific sites and supercoiling on looping of DNA by Fis should be studied in future experiments.

This research was supported by NSF Grant No. DMR0203963, No. MCB-0240998, and No. PHY-0445565, and by USPHS Grant No. GM38509.
[1] R. C. Johnson, L. M. Johnson, J. W. Schmidt, and J.F. Gardner,in The Bacterial Chromosome, edited by N.P. Higgins (ASM, Washington, D.C., 2004), p 65.

[2] C. A. Ball, R. Osuna, K. C. Ferguson, and R. C. Johnson, J. Bacteriol. 174, 8043 (1992).

[3] B. M. Ali, R. Amit, I. Braslavsky, A. B. Oppenheim, O. Gileadi, and J. Stavans, Proc. Natl. Acad. Sci. U.S.A. 98, 10658 (2001).

[4] J. Yan and J. F. Marko, Phys. Rev. E 68, 011905 (2003).

[5] N. P. Higgins, S. Deng, Z. Pang, R. A. Stein, K. Champion, and D. Manna, in The Bacterial Chromosome, edited by N. P. Higgins (ASM, Washington, D.C., 2004), p. 133.

[6] L. Postow, C. D. Hardy, J. Arsuaga, and N. R. Cozzarelli, Genes Dev. 18, 1766 (2004).

[7] C. G. Baumann, V.A. Bloomfield, S. B. Smith, C. Bustamante, M.D. Wang, and S. M. Block, Biophys. J. 78, 1965 (2000).

[8] S. B. Smith, L. Finzi, and C. Bustamante, Science 258, 1122 (1992).

[9] D. Skoko, B. Wong, R. C. Johnson, and J. F. Marko, Biochemistry 43, 13867 (2004).

[10] J.F. Allemand, D. Bensimon, and V. Croquette, Curr. Opin. Struct. Biol. 13, 266 (2003).

[11] G. Charvin, T. R. Strick, D. Bensimon, and V. Croquette, Biophys. J. 89, 384 (2005).

[12] J.F. Marko, and E. D. Siggia, Macromolecules 28, 8759 (1995).

[13] C. Bustamante, S. B. Smith, J. Liphardt, and D. Smith, Curr. Opin. Struct. Biol. 10, 279 (2000).

[14] J. van Noort, S. Verbrugge, N. Goosen, C. Dekker, and R. T. Dame, Proc. Natl. Acad. Sci. U.S.A. 101, 6969 (2004).

[15] C. Q. Pan, R. C. Johnson, and D. S. Sigman, Biochemistry 35, 4326 (1996).

[16] D. Yoo and R. C. Johnson (unpublished).

[17] J. F. Marko and E. D. Siggia, Biophys. J. 73, 2173 (1997).

[18] S. Sankararaman and J. F. Marko, Phys. Rev. E 71, 021911 (2005).

[19] J. Yan, D. Skoko, and J.F. Marko, Phys. Rev. E 70, 011905 (2004).

[20] R. Schneider, R. Lurz, G. Luder, C. Tolksdorf, A. Travers, and G. Muskhelishvili, Nucleic Acids Res. 29, 5107 (2001).

[21] J. Shimada and H. Yamakawa, Macromolecules 17, 689 (1984).

[22] S. Dixit, M. Singh-Zocchi, J. Hanne, and G. Zocchi, Phys. Rev. Lett. 94, 118101 (2005).

[23] S. Sankararaman and J. F. Marko, Phys. Rev. Lett. 95, 078104 (2005).

[24] M. McCauley, P. R. Hardwidge, L. J. Maher, and M. C. Williams, Biophys. J. 89, 353 (2005).

[25] R. A. Stein, S. Deng, and N. P. Higgins, Mol. Microbiol. 56, 1049 (2005).

[26] A. Travers and G. Muskhelishvili, J. Mol. Biol. 279, 1027 (1998).

[27] J.F. Thompson, L. Moitoso de Vargas, C. Koch, R. Kahmann, and A. Landy, Cell 50, 901 (1987).

[28] A. V. Vologodskii and N. R. Cozzarelli, Annu. Rev. Biophys. Biomol. Struct. 23, 609 (1994). 